Portland State University

PDXScholar

Comparison of Monowave and Polywave Transmission and Curing Profile thru Various Composites

Anna Kolpakova

Portland State University

Follow this and additional works at: https://pdxscholar.library.pdx.edu/honorstheses Let us know how access to this document benefits you.

Recommended Citation

Kolpakova, Anna, "Comparison of Monowave and Polywave Transmission and Curing Profile thru Various Composites" (2015). University Honors Theses. Paper 170.

https://doi.org/10.15760/honors.173

This Thesis is brought to you for free and open access. It has been accepted for inclusion in University Honors Theses by an authorized administrator of PDXScholar. Please contact us if we can make this document more accessible: pdxscholar@pdx.edu. 


\title{
Comparison of Monowave and Polywave Transmission and Curing Profile thru Various Composites
}

\author{
By \\ Anna Kolpakova
}

An undergraduate honors thesis submitted in partial fulfillment of the

Requirements for the degree of

Bachelor of Science

in

University Honors

and

Biochemistry

Thesis Advisor

Jack Ferracane PhD

Portland State University

May 30 ${ }^{\text {th }}, 2015$ 


\title{
Contents
}

\author{
Abstract 3
}

1. Introduction 3

1.1 What are composites?...................

1.2 Previous Research ....................4

2. Methods and Materials 6

2.1 Dental Light Curing Unit Characterization .......6 6

2.2 Composite Characterization .............. 7

2. 3 Sample Prep and Transmission Measurements ... 7

3. Results 8

3.1 Tables and Figures. .................

2.2 Description........................

4. Discussion. ......................... 11

Acknowledgements....................... 13

Bibliography..........................13 


\begin{abstract}
Objective: To compare the light-transmission and spectral output of a polywave and monowave LCU thru two different composites at varying thicknesses.

Methods: For irradiance measurements two bulk fill composites $(n=3)$ containing different photoinitiators: CQ-based (SonicFill, Kerr) and TPO and CQ-based (Tetric EvoCeram, Ivoclar Vivadent) were prepared in Delrin molds of varying thicknesses (1mm, 2mm, and 3mm). A Resin Calibrator (MARC Resin Calibrator, BlueLight Analytics) was used to determine irradiance and spectrum of two different curing lights as the samples were cured for 20 seconds: a polywave (Bluephase G2, Ivoclar Vivadent) and a monowave (SmartLite Focus, DENTSPLY) LCUs. Results: The polywave LCU had two peaks at about 407nm (violet) and at 455.58nm (blue) and the monowave had one peak at 473.04nm (blue). For both LCUs, the TPOand CQ-based composite had a higher percentage of irradiance transmitted thru all three thicknesses when compared to the CQ-based composite. When comparing curing lights the monowave had a higher percent transmittance thru both of the composites compared to the polywave unit.
\end{abstract}

\title{
1.Introduction
}

\subsection{What are dental composites?}

Every year thousands of people in the U.S visit the dentist to get fillings done. Generally these are either the "silver" colored amalgam fillings or the tooth colored resinbased dental composites (RBC). There are two different classes of RBCs: incremental, which are cured in $2 \mathrm{~mm}$ increments and bulk fill which have an average curing depth of up to $4 \mathrm{~mm} .{ }^{7,9}$ For cosmetic reasons the more expensive RBCs are preferred to the amalgams by patients. However, RBC's are less strong and on average have a lifespan that is 2-4 years shorter than amalgams. ${ }^{14}$ This leads to financial and health implications for patients because the patients end up paying more to replace their filling and their teeth are not as well protected against the enzymatic and bacterial environment of the mouth. A possible reason for premature failure of the $\mathrm{RBC}$ is inadequate cure of the polymer. ${ }^{5}$

Curing is the polymerization of the resin composite thru the process of photopolymerization. This happens when the malleable resin is subjected to irradiation from a light source resulting in the conversion of the resin into an insoluble polymer ${ }^{8,15} \mathrm{In}$ order for this reaction to happen the resin must contain a photoinitiator. There are two types of photoinitiators. A type I photo-initiator is a molecule that will generate free radicals by dissociating after absorbing light from a specific range of wavelengths. ${ }^{15} \mathrm{~A}$ type II generates an excited state after it absorbs the necessary wavelengths of light. In this excited state it reacts with a tertiary amine co-initiator to generate the free radicals. ${ }^{15}$ Different photo-initiators absorb light at different wavelengths. The formation of free radicals from the excited state complex leads to the crosslinking of monomers in the resin resulting in the formation of the polymer ${ }^{8}$ Two popular photoinitiators used in dental composites are camphorquinone (CQ) and monoacylphosphine oxide (TPO). CQ is a bright yellow type II photo initiator that absorbs light in the visible spectrum at $467 \mathrm{~nm}$ and in the UV region of $200-300 \mathrm{~nm} .^{8}$ TPO is light yellow type I photoinitiator and 
absorbs from approximately 295-390nm. ${ }^{2}$ The two photoinitiators will either be used by themselves or together in different concentrations along with their co-initiators. These concentrations will determine the degree of cure in the composite and the color of the composite. ${ }^{12}$ The bright yellow color of CQ does not get fully bleached when it is photo activated which has lead manufacturers to add other, less chromatic photo initiators such as TPO into the resins to reduce the yellowing effect.

In order for the photoinitiator to be activated it must be subjected to light from a source. ${ }^{2,8,15}$ The light source used to cure composites is usually an LED-based light curing unit (LCU). The output wavelength of monowave LCUs is generally in the visible range of $445 \mathrm{~nm}$ to $480 \mathrm{~nm}$. ${ }^{14}$ To get maximum cure efficiency the output wavelength from the LED is usually matched to the absorption wavelength of the photo initiator, in this case CQ which absorbs at 467nm . ${ }^{8,11,12}$ At wavelengths around 460nm the TPO is not as efficiently activated, resulting in a lower degree of cure, because it absorbs at a lower wavelength range of $295 \mathrm{~nm}-390 \mathrm{~nm} .{ }^{2,11}$ The mismatch of LCU output to composite is a common problem because composite composition and choice of photoinitiators varies amongst manufacturers and products. In order to efficiently cure a wide range of composites with added photoinitiators such as TPO, manufacturers of LCUs have introduced new polywave LCU . 1,10,14 Monowave LCUs have LED chips that all output at approximately the same wavelength. Even so the light beam from the LCU has an inhomogeneous irradiance and power output. ${ }^{10,14}$

The new polywaves combine LED chips that output near the camphorquinone range and the 400-410nm range, which is close to the absorption range of TPO, allowing it to be more efficiently activated. ${ }^{1,6,7,10,11,15}$ However, the introduction of new wavelength outputs further compounds the inhomogeneity of irradiance and power of the light beam. Recent research indicates that this spectral and power inconsistency can possibly lead to uneven cure of the composite. ${ }^{1,11,14}$ In order to achieve thorough polymerization all parts of the restoration must be subjected to optimal intensity of light at the necessary wavelength for the photoinitiator. ${ }^{1,3,5,10,11,12,13,14,16}$ Inhomogeneous light at the incorrect wavelength or intensity may result in insufficient and uneven polymerization. ${ }^{1,3,5,10,11,12,13,14,16}$ For thicker bulk fill composites this is especially important because there is more resin for the light to travel through before reaching the bottom surface of the composite. This has further implications because an uneven or inadequate polymerization of the resin composite may lead to premature failure of the restoration due to problems such as increased wear and marginal breakdown. ${ }^{5}$ The purpose of this study is to compare the amount of light and curing profile from a monowave and polywave LCU reaching the bottom of two different bulk fill composites at different thicknesses .

\subsection{Previous Research}

As stated previously, it has been shown that the beam profile of both monowave and polywave LCUs is not homogenous. ${ }^{1,10,14}$ A previous study used a laser beam profiler to measure the irradiance distribution across the light guide tip of a monowave Elipar S10 (3M ESPE), the polywaves Bluephase Style (Ivoclar Vivadent), and SmartLite Max (DENTSPLY). ${ }^{10}$ In a later study by the same researchers, bandpass filters were introduced to measure the irradiance distribution at the two emitted wavelengths of $409 \mathrm{~nm}$ and $456 \mathrm{~nm}$ for the Bluephase Style. ${ }^{14}$ In both studies the camera image from the 
laser beam profiler showed distinct hotspots of irradiance directly corresponding to the location of the LED chips for both the Bluephase Style and the SmartLite Max indicating that different areas of the composite were receiving different amounts of irradiance. ${ }^{10,14}$ The bandpass filters showed exactly the same results indicating that the Bluephase Style had a very non-uniform beam profile. The average irradiance in one study was calculated to be $1055 \pm 8 \mathrm{~mW} / \mathrm{cm} 2$ (the other study calculated it at $1056 \pm 7 \mathrm{~mW} / \mathrm{cm}^{2}$ ) and the power was determined to be $31.7 \mathrm{~J} / \mathrm{cm}^{2}$ after a 30 second cure by the Bluephase Style. However, the median of irradiance at 45 points within the light tip was determined to be 1013 $\mathrm{mW} / \mathrm{cm}^{2}{ }^{14}$ The monowave Elipar had a much more homogenous output with beam homogeneity factors of $49 \%$ compared to the Bluephas Style which only had 2\%.

Furthermore, both studies used an integrating sphere to measure the spectral emission of the LCUs. The monowave Elipar S10 delivered a range of 430nm-490nm evenly across its light tip. However, it had uneven power output with higher spectral power towards the middle of the monowave which decreased towards the edges. ${ }^{10}$ The Bluephase Style had both non-uniform spectral emission and non-uniform power. This resulted in spots only emitting light at $409 \mathrm{~nm}$ or $456 \mathrm{~nm} .{ }^{10,14}$ In the center of the Bluephase Style both 409nm and 456nm wavelengths are present however their spectral power is much less than in the hotspots directly corresponding to the position of the LEDs. This means that based on the orientation of the LCU different areas of RBC will receive different amounts of power at different wavelengths. Thus, the objective of the later study was to determine what was the correlation between the inhomogeneous beam profile and the microhardness of the top and bottom surfaces of four different composites.

Microhardness maps of the top and bottom surfaces of the composites showed a positive correlation between the inhomogeneity of the beam profile and the inhomogeneity in microhardness of the composite. ${ }^{10}$ Since inhomogeneity was seen at the bottom along with the top this indicates that there could possibly be inhomogeneity throughout the RBC. Furthermore, the correlation between microhardness and beam inhomogeneity was stronger on the bottom than the top. ${ }^{14}$ This could possibly indicate that with depth the cure of the composite became more inhomogeneous. For composites that only contained CQ this correlation decreased when curing time was increased. However, for composites that contained both CQ and TPO the effect was the opposite. A possible reason for this provided by the study was Rayleigh scattering, the lower wavelengths $(409 \mathrm{~nm})$ needed to activate the TPO would have scattered more, resulting in a longer curing time needed for more of them to reach the photoinitiators towards the bottom of the RBC. The results of this study are in direct agreement with another study published in 2007 by Arikawa, et al.

An acrylic optical fiber was used to measure the light intensity of different curing lights (including one LED) at various points on the light guide tip. The light intensity was directly correlated to microhardness values on the top surface of the composite. The composite used Camphorquinone as the photoinitiator. As previously seen the intensity of light across the guide tip varied dramatically. The inhomogeneity of microhardness on the surface of the composite was found to be less than the inhomogeneity in the intensity due to possible effects of increased power output. However, even with the LCU exhibiting the highest power output, inhomogeneity in microhardness was present on the surface of the composite. The overall results of these studies indicate that different parts 
of the composite will be subjected to different amounts of irradiance and different wavelengths resulting in inhomogeneous cure of the composite.

This is important because for composites with camphorquinone as the photoinitiator, both the wavelength and quantity of light reaching it affect its amount of cure. ${ }^{8,12,15}$ A study on the effects of wavelength on the composites polymerization determined that during the beginning of cure the absorption of wavelengths had the largest effect. ${ }^{12} \mathrm{~A}$ wavelength of $470 \mathrm{~nm}$ was the most efficient, however wavelengths in the 450-490nm range worked well especially with increasing exposure time. The absorption spectrum collected by the study showed the maximum absorption for CQ to be at $467 \mathrm{~nm}$. The maximum degree of conversion for all irradiance times was found to be at 470nm directly corresponding to the absorption wavelength of CQ. Furthermore it was determined that wavelengths beyond 490nm had a harder time exciting CQ than wavelengths below 470nm. After 30 seconds of cure time the DC at wavelengths just below $470 \mathrm{~nm}$ began to reach DC seen at $470 \mathrm{~nm}$. The DC continued to increase with exposure time indicating that in the $450 \mathrm{~nm}-490 \mathrm{~nm}$ range the exposure amount is more important than actual wavelengths to the degree of conversion. This was supported by a further experiment where exposure was kept constant and wavelength was varied. ${ }^{13,12}$ The DC of conversion for exposure times beyond 30 seconds was approximately the same for wavelengths in the 450-490nm range.

This effect of increasing curing efficiency of the LCU by increasing exposure time was supported by a study done by David et al. ${ }^{3}$ They measured microhardness at the top and bottom surface of their samples. The microhardness on the bottom surface of the sample was significantly increased when the curing time was increased to 40 seconds. Increasing the curing time beyond the 40 seconds did not result in a considerable effect on the microhardness. A hypothesis brought up for further studies was that light focusing of the LCU beam would result in less light diffusion deeper into the composites and thus would require a longer exposure time for sufficient polymerization. Another hypothesis presented is that composites with quick curing at the top surface would have larger scattering and absorption affects resulting in less light reaching the bottom of the restoration.

In all, the studies available show that the quantity and quality of the light reaching the photoinitiators in all parts of the RBC is important for the composite to be adequately cured.

\section{Methods and Materials}

\subsection{Dental Light Curing Unit Characterization}

The two dental units being tested are the polywave Bluephase G2 by Ivoclar Vivadent and the monowave SmartLite Focus by Dentsply. The Bluephase G2 is a polywave with four LEDs, three of which have the same output. This results in a broad output spectrum of $385 \mathrm{~nm}$ to $515 \mathrm{~nm}$ with two main irradiance peaks. ${ }^{6}$ The minor peak is found at 410nm (only one of the LEDs) and is meant to overlap with the absorption spectrum of the photoinitiator Lucirin TPO. ${ }^{7}$ The major peak corresponding to the other three LED lights is indicated to be at 470nm, overlapping the absorption spectrum of Camphorquinone which is the major photo initiator used in composites. ${ }^{7}$ According to the manufacturer the Bluephase $\mathrm{G} 2$ has a light output of $1,100 \pm 10 \% \mathrm{~mW} / \mathrm{cm}^{2}$ with an 
average irradiance of $1,066 \mathrm{~mW} / \mathrm{Cm}^{2}{ }^{6}$ The light curing unit has three different modes: High, Low, and Soft. The high program was chosen for this study in order to keep the Bluephase output comparable to the Smartlite Focus and to determine the maximum light going thru the composite. The time chosen for the curing cycle was 20 seconds due to manufacturer recommendations for both composites and the fact that the SmartLite Focus can only be set to cure for twenty seconds.

The SmartLite Focus is a monowave with only one curing cycle with consistent irradiance for 20 seconds. ${ }^{4}$. Unlike the Bluephase G2 it has only one LED and delivers light to the composite in a collimated beam supposedly resulting in less scatter and deeper depth of cure (up to $8 \mathrm{~mm}$ ). The emission spectrum of the LED is directed towards the absorption spectrum of camphorquinone with an emission range from around 380nm$500 \mathrm{~nm}$ (around the same as the G2) with one peak at around 465nm . There is no second peak meant to overlap the absorption spectrum of Lucirin TPO. According to the manufacturer SmartLite has a $79.7 \%$ efficiency of initiating camphorquinone in relation to the 70.4-77.8\% efficiency of the Bluephase G2. ${ }^{4}$ Furthermore, its beam is more evenly distributed than the polywave. ${ }^{4}$ The light output of the SmartLite is approximately at $1,100 \pm 10 \%$ per studies done by the manufacturer, which is the same as the G2 .

\subsection{Composite Characterization:}

The two composites chosen for this study are: Tetric EvoCeram by Ivoclar Vivadent and SonicFill by Kerr. Both composites are bulk fill and designed for deep restorations. In order to prevent any additional effect from color, the colors of the composites are comparable, Tetric is IVB and SonicFill is A1. Per manufacturers, SonicFill has a cure depth of $5 \mathrm{~mm}$ while Tetric's is $4 \mathrm{~mm} .^{7,9}$ The manufacturer recommends the use of an LED curing light from the Bluephase family to cure Tetric. ${ }^{7}$ There is no recommended curing light for SonicFill. Furthermore, the manufacturer does not list the photoinitiators used in the composite, the only thing mentioned is that the amount of photoinitiators used is larger than in other composites ${ }^{9}$ Hence for this study it is assumed that the only photoinitiator used in SonicFill is CQ, which is the major photoinitiator used in other composites. The Tetric EvoCeram composite has three photoinitiators present in it: camphorquinone with a peak absorbance at 470nm, Lucirin TPO (an acyl phosphine oxide) with a peak absorbance of about $385 \mathrm{~nm}$, and the company's new patented photoinitiator called Ivocerin which absorbs the strongest at $408 \mathrm{~nm} .{ }^{7}$ The manufacturer recommends the use of the polywave to cure the composite due to the presence of Lucirin TPO and Ivocerin, which absorb at wavelengths much less than camphorquinone.

\subsection{Sample Preparation and Transmission Measurements}

The composite samples were cured in Delrin discs $(\mathrm{d}=6 \mathrm{~mm}$ with a $1 \mathrm{~mm}, 2 \mathrm{~mm}$, and $3 \mathrm{~mm}$ thickness) that were placed onto the bottom sensors of the Marc Resin Calibrator (Blue Light Analytics, Halifax, Canada). A Mylar strip was placed on top of the Delrin disc and a glass slide was used to squeeze out any excess material. The Bluephase G2 or the SmartLite Focus was used to irradiate all samples for 20 seconds at the high mode. Baseline transmission and irradiance measurements were taken for both LCU's thru all three thicknesses of the Delrin discs without the composites present. 


\section{Results}

\subsection{Tables and Figures}

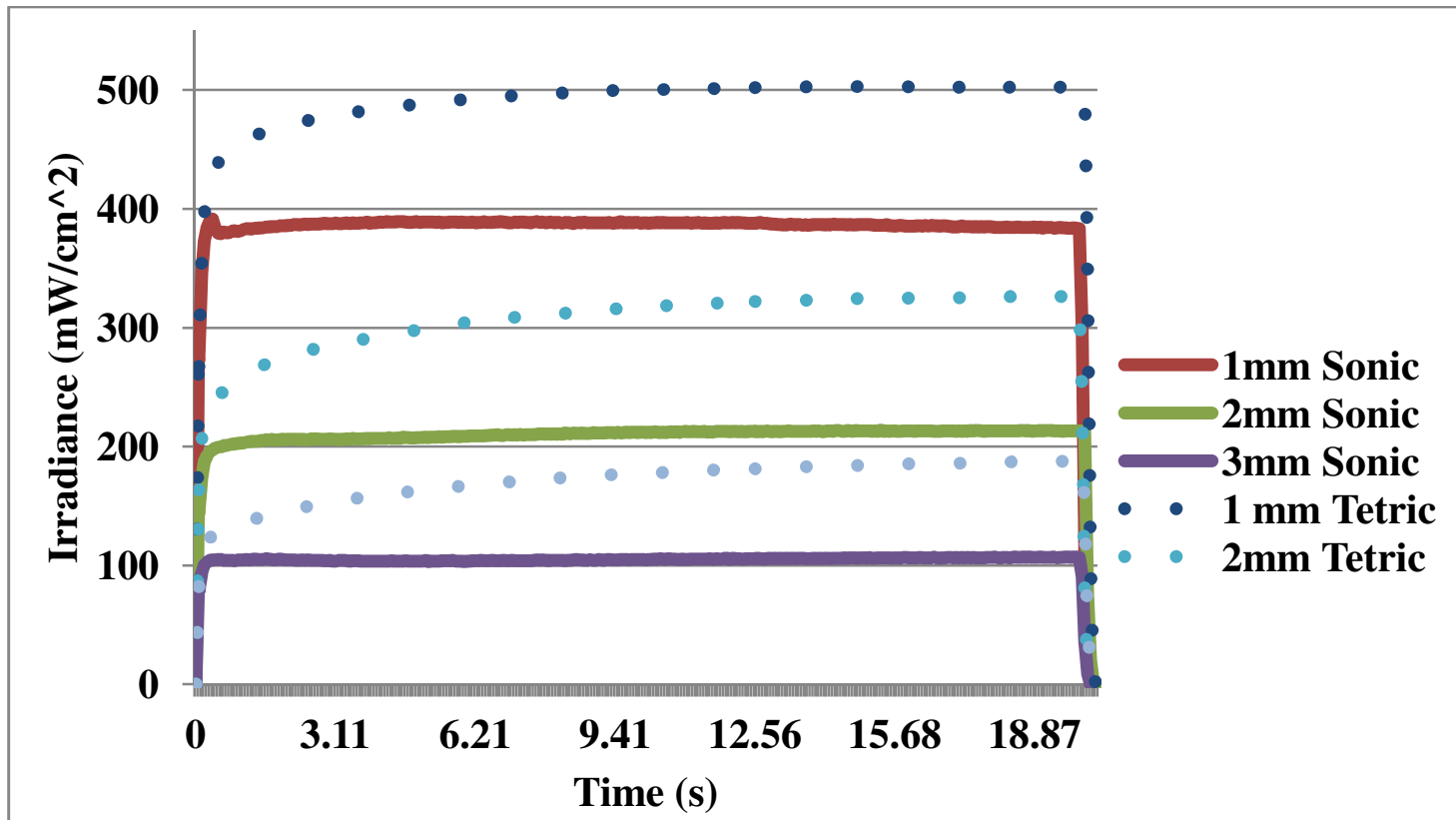

Figure 1: Irradiance output during 20 seconds of cure time of the Bluephase G2 while curing the SonicFill and Tetric thru different thicknesses (1mm, 2mm, and $3 \mathrm{~mm})$.

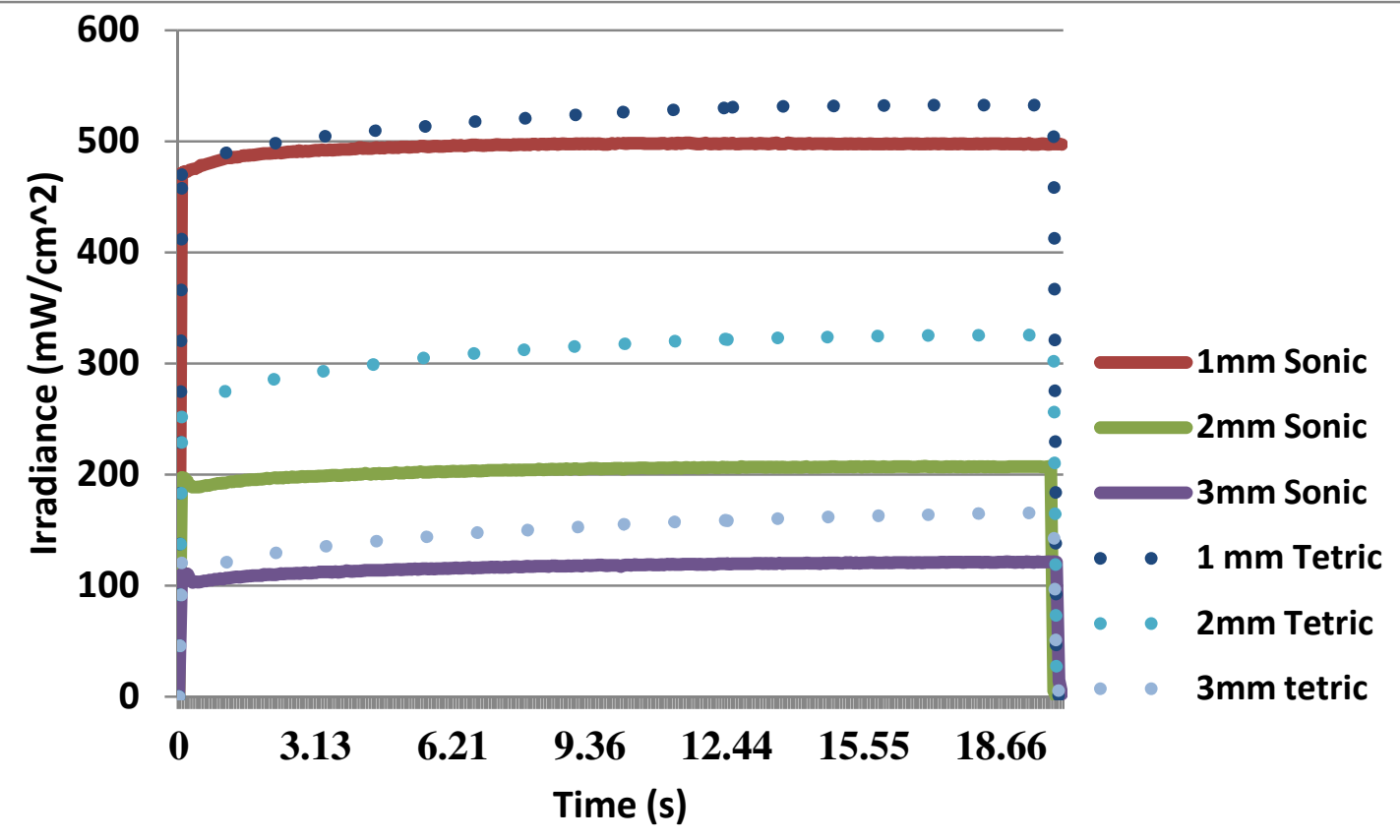

Figure 2: Irradiance output during 20 seconds of cure time of the SmartLite Focus while curing the SonicFill and Tetric thru different thicknesses (1mm, $2 \mathrm{~mm}$, and $3 \mathrm{~mm}$ ). 
Table 1: Shows the irradiance and power transmitted by the polywave and the monowave thru the CQ and CQ plus TPO containing composites.

\begin{tabular}{|c|c|c|c|c|c|}
\hline Bluephase & $\begin{array}{l}\text { Average } \\
\text { Irradiance } \\
(\mathrm{mW} / \mathrm{cm})\end{array}$ & $\begin{array}{c}\% \text { of } \\
\text { irradiance } \\
\text { transmitted }\end{array}$ & $\begin{array}{l}\text { Difference in \% } \\
\text { transmittance } \\
\text { between lights }\end{array}$ & $\begin{array}{c}\text { Total } \\
\left(\mathrm{J} / \mathrm{cm}^{2}\right)\end{array}$ & $\begin{array}{c}\% \text { Power } \\
\text { Transmitted }\end{array}$ \\
\hline Blank 1mm & 1461.27 & & & 29.401 & \\
\hline Blank 2mm & 1556.126 & & & 31.275 & \\
\hline Blank 3mm & 1572.644 & & & 31.667 & \\
\hline Tetric $1 \mathrm{~mm}$ & 488.186 & 33.41 & & 9.799 & 33.33 \\
\hline Tetric $2 \mathrm{~mm}$ & 304.59 & 19.57 & & 6.126 & 19.59 \\
\hline Tetric 3mm & 169.855 & 10.80 & & 3.416 & 10.79 \\
\hline Sonic $1 \mathrm{~mm}$ & 382.904 & 26.20 & & 7.703 & 26.20 \\
\hline Sonic $2 \mathrm{~mm}$ & 208.254 & 13.38 & & 4.185 & 13.38 \\
\hline Sonic $3 \mathrm{~mm}$ & 104.468 & 6.64 & & 2.092 & 6.61 \\
\hline \multicolumn{6}{|l|}{ SmartLite } \\
\hline Blank 1mm & 1131.189 & & & 22.727 & \\
\hline Blank 2mm & 1079.617 & & & 21.781 & \\
\hline Blank 3mm & 1138.894 & & & 22.988 & \\
\hline Tetric $1 \mathrm{~mm}$ & 516.877 & 45.69 & 12.28 & 10.387 & 45.70 \\
\hline Tetric $2 \mathrm{~mm}$ & 326.22 & 30.22 & 10.64 & 6.237 & 28.64 \\
\hline Tetric $3 \mathrm{~mm}$ & 149.59 & 13.13 & 2.33 & 3.01 & 13.09 \\
\hline Sonic 1mm & 492.631 & 43.55 & 17.35 & 9.898 & 43.55 \\
\hline Sonic $2 \mathrm{~mm}$ & 202.94 & 18.80 & 5.41 & 4.09 & 18.78 \\
\hline Sonic $3 \mathrm{~mm}$ & 116.207 & 10.20 & 3.56 & 2.347 & 10.21 \\
\hline
\end{tabular}

\subsection{Description}

Both figure 1 and figure 2 are a visual representation of the irradiance transmitted thru the composite over twenty seconds. For both the monowave and the polywave they show that the irradiance increases to its maximum point and remains there for the rest of the twenty seconds indicating a consistent output from the LCU. However, this increase is different for the composites. In both figure 1 and 2 the irradiance graph for the CQbased composite is mostly flat, increasing to its maximum point almost immediately and staying there the rest of the twenty seconds. The CQ and TPO-based composite graphs have more of a curve to them, taking longer to reach their "maximum" irradiance that continues to increase slowly after about 9 seconds. Furthermore, for both the polywave and the monowave, the CQ- and TPO-based composite has a higher irradiance at all thicknesses. This is supported by Table 1, which shows that the CQ- and TPO-based composite had a higher percent transmittance than the CQ-based composite when cured by both the LCUs. The higher transmittance and slow increase of the maximum irradiance should result in a higher total power going thru the composite. 


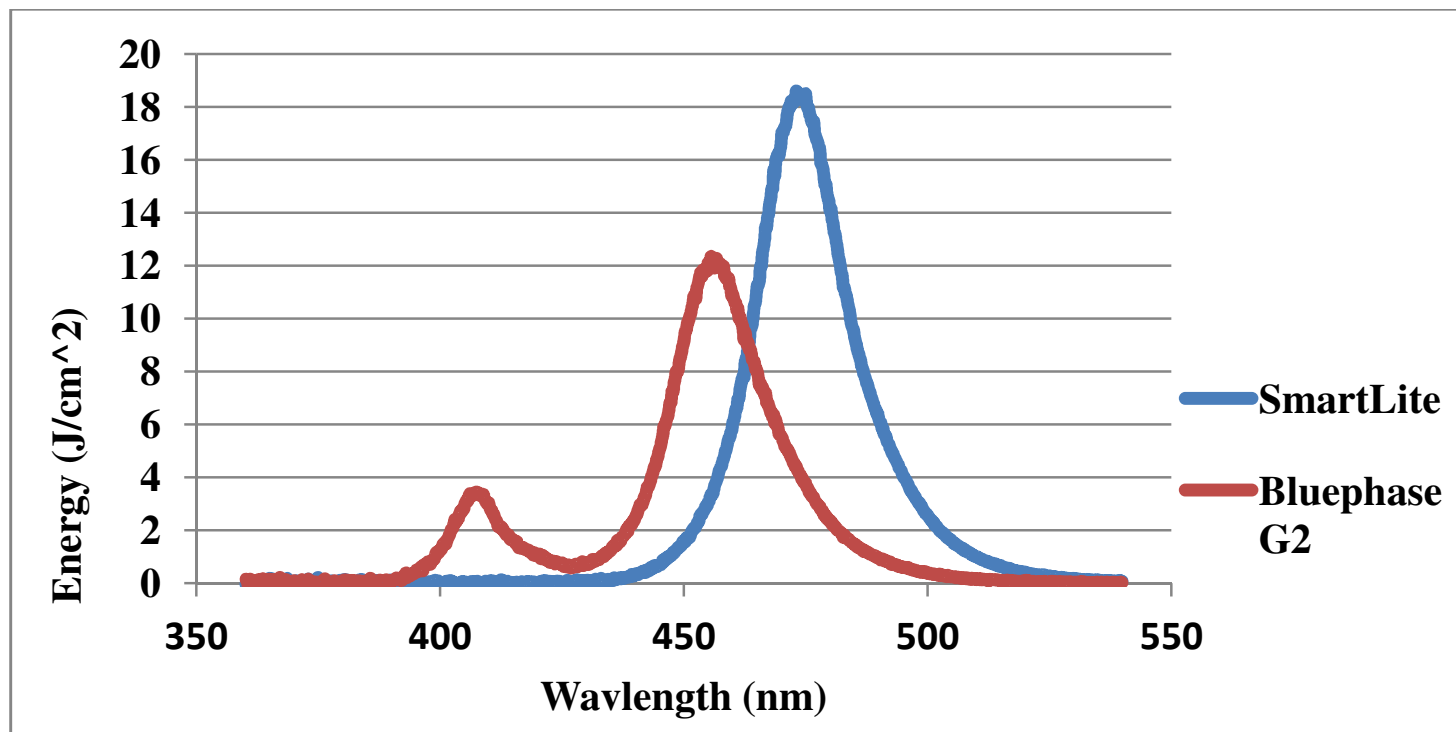

Figure 3: Shows the comparison between the Bluephase G2 and SmartLite Focus spectra for $1 \mathrm{~mm}$ SonicFill. Bluephase G2 has two peaks: one at $406.08 \mathrm{~nm}$ and one at $455.58 \mathrm{~nm}$. SmartLite Focus has one peak at $473.04 \mathrm{~nm}$.

Table 2: Shows the power output in J/ $\mathrm{cm}^{2}$ for the Bluephase and Smartlite in the TPO absorption rangde of $340-420 \mathrm{~nm}$ and the CQ absorption range of $420-540 \mathrm{~nm}$.

\begin{tabular}{|c|r|r|}
\hline Bluephase & $\begin{array}{r}\text { 340nm- } \\
\text { 420nm }\end{array}$ & $\begin{array}{r}420- \\
540 \mathrm{~nm}\end{array}$ \\
\hline Blank $1 \mathrm{~mm}$ & 6.587 & 22.814 \\
\hline Blank $2 \mathrm{~mm}$ & 7.724 & 23.55 \\
\hline Blank $3 \mathrm{~mm}$ & 7.288 & 24.38 \\
\hline Tetric $1 \mathrm{~mm}$ & 0.883 & 8.915 \\
\hline Tetric $2 \mathrm{~mm}$ & 0.246 & 5.881 \\
\hline Tetric $3 \mathrm{~mm}$ & 0.065 & 3.351 \\
\hline Sonic $1 \mathrm{~mm}$ & 1.012 & 6.691 \\
\hline Sonic $2 \mathrm{~mm}$ & 0.27 & 3.915 \\
\hline Sonic $3 \mathrm{~mm}$ & 0.096 & 1.997 \\
\hline SmartLite & & \\
\hline Blank $1 \mathrm{~mm}$ & 0.065 & 22.662 \\
\hline Blank $2 \mathrm{~mm}$ & 0.074 & 21.707 \\
\hline Blank $3 \mathrm{~mm}$ & 0.079 & 22.909 \\
\hline Tetric $1 \mathrm{~mm}$ & 0.024 & 10.363 \\
\hline Tetric $2 \mathrm{~mm}$ & 0.023 & 6.214 \\
\hline Tetric $3 \mathrm{~mm}$ & 0.013 & 2.997 \\
\hline Sonic $1 \mathrm{~mm}$ & 0.029 & 9.869 \\
\hline Sonic $2 \mathrm{~mm}$ & 0.012 & 4.078 \\
\hline Sonic $3 \mathrm{~mm}$ & 0.009 & 2.337 \\
\hline
\end{tabular}

This is shown in Table 1, for both the polywave and the monowave the CQ and TPO- based composite has higher total power than the CQ-based composite .

In figure 1, the irradiance values between the two composites at the same thickness are fairly spaced out. The difference in irradiance is around $100 \mathrm{~mW} / \mathrm{cm}^{2}$. In figure 2 , which shows the irradiance values from the monowave, the $1 \mathrm{~mm}$ and $3 \mathrm{~mm}$ values are much closer together. Indicating that at those thicknesses the two composites are getting similar amounts of irradiance. This is shown in Table 1 where the difference in percent transmitted between the composites is around $2 \%$ for both the $1 \mathrm{~mm}$ and $3 \mathrm{~mm}$ thickness. However, the $2 \mathrm{~mm}$ thickness has a large percent difference in both figure 1 and 2 . In figure 2 , the difference in percent transmitted is $11.42 \%$ as seen in Table 1 .

Table 1 shows that the monowave 
had a higher percent transmittance for both composites than the polywave. The highest difference between the two LCUs was seen in the $1 \mathrm{~mm}$ thickness of the CQ-based composite. For both composites as the thickness of the composite increased the difference in transmittance between the two lights became less noticeable. However, in the CQ and TPO-based composite the difference between the two LCUs was still substantial at $2 \mathrm{~mm}$ at $10.64 \%$.

Figure 3 is the emission spectra of the polywave and the monowave. Even though the figure only shows the emission spectra thru the $1 \mathrm{~mm}$ CQ-based composite, the rest of the emission spectra for the other thicknesses and for the CQ and TPO-based composite showed differences only in the height of the peaks,. Figure 3 shows the polywave light had two peaks at $407 \mathrm{~nm}$ and at $455.58 \mathrm{~nm}$ and the monowave only had one peak at $473.04 \mathrm{~nm}$. Since the polywave had a second peak, its peak at $455.58 \mathrm{~nm}$ is smaller than the one the monowave has at $473.04 \mathrm{~nm}$.

Table 2 shows the power transmitted in the wavelength ranges for Lucirin TPO (340nm-420nm) and for camphorquinone (420nm-520nm). The monowave shows barely any power in the 340-420nm range thru both composites. This means that all of its power is found in the CQ range, which is why in figure 3 the peak has a greater height than the polywave peak. The polywave shows some power in the TPO range. However, as the thickness of both composites increases to $3 \mathrm{~mm}$, the amount of power drops dramatically. The polywave exhibits less power at all thicknesses in the CQ region than the monowave, due to one its LEDs outputting light in the violet range. As seen in table 1, the CQ and TPO-based composite shows more power being transmitted in the CQ range for both the polywave and monowave LCU. However, in the TPO range the CQbased composite shows more power being transmitted using the polywave. With the monowave both composites have approximately the same power transmittance in the TPO range.

\section{Discussion}

In order to take light scattering into account, the composite measurements were compared to the blank measurements of the same height. Since the monowave curing light is a collimated beam, as the distance increases the average irradiance should not change dramatically. Thus it makes sense that the average irradiance for the $1 \mathrm{~mm}$ and $3 \mathrm{~mm}$ are within $7 \mathrm{~mW} / \mathrm{cm}^{2}$ of each other. More data would be needed to determine whether this pattern is actually true especially because of the lower irradiance seen at $2 \mathrm{~mm}$. For the polywave the average irradiance increased with the Delrin disc thickness.

For both the polywave and the monowave the CQ and TPO-based composite had a higher percentage of irradiance transmitted thru all three Delrin disc thicknesses when compared to the CQ-based composite. The same result was seen with the total power, which is directly related to the amount of irradiance going thru. This is somewhat surprising because the SonicFill cure depth is advertised by the manufacturer to be $1 \mathrm{~mm}$ larger than the Tetric's indicating that the percent transmittance thru the CQ-based composite should be higher in order to reach the photoinitiators deeper in the composite. Purely based on the amount of irradiance going through the CQ-and-TPO based composite should have better cure efficiency with increasing thickness. Furthermore, table 2 shows that the CQ and TPO-based composite has more power being transmitted in 
the CQ range than just the CQ-based composite, indicating that based on the amount of light going through the CQ should be more efficiently activated in the CQ and TPObased composite. Even though the CQ-based composite had more power being transmitted in the TPO range, the assumption made at the beginning of this study was that there was no TPO in the composite so this should have no considerable effect on the curing efficiency of the composite.

When comparing curing lights the monowave had a higher percent transmittance thru both of the composites. It has a collimated beam, which may account for the higher percent of irradiance going thru and not getting lost to the sides of the composite such as would happen with the polywave. For both lights the difference in transmittance between different thicknesses went down as the thickness of the composite increased. This indicates that the thicker the composite the less light will reach the photoinitiators on the bottom which should result in a lower level of cure the deeper you go into the composite. This is something that should be supported by the degree of conversion measurements taken by an FTIR.

In terms of the spectral output, the polywave had two peaks at about $407 \mathrm{~nm}$ and at $455.58 \mathrm{~nm}$. The monowave had only one peak at $473.04 \mathrm{~nm}$. The CQ and TPO-based composite has three photoinitiators present in it: CQ (470nm absorbance peak) Lucirin TPO (385nm), and Ivocerin (408nm) while the CQ-based composite only has CQ. ${ }^{7}$ The polywave has irradiance peaks overlapping all three of the photoinitiators. However, previous studies have shown that CQ is activated most efficiently at $470 \mathrm{~nm}$. Between the polywave and the monowave, the monowave should be better suited for activating the CQ in both composites because its peak irradiance is at 473.04 and the polywave peak is at 455.58. ${ }^{12}$ The same study showed that a wavelength of less than $470 \mathrm{~nm}$ could efficiently cure CQ only if the spectral output is increased. This is not the case with the polywave. Some of the power being delivered is in the violet range and thus scatters more. This means that less power is seen in the CQ range, which is what we see in table 2. In both composites the monowave should activate the CQ more efficiently because its output matches the CQ peak absorption and has higher power in the 420-540nm range.

However, the monowave may not be very efficient for the Lucirin TPO and Ivocerin whose absorbance peaks are located much lower at $385 \mathrm{~nm}$ and $408 \mathrm{~nm}$. With a second peak at $407 \mathrm{~nm}$, the polywave should react better with the two photoinitiators. However, in table 2 it is shown that the amount of energy transmitted by the polywave in the 340-420nm range, decreases dramatically with increasing thickness which may be due to higher scattering of the lower wavelengths making it harder for them to reach the deeper photoinitiators. This is important because both composites are bulk fill and will be used for deep restorations. Thus even though the monowave does not have a peak in the TPO absorption range, it is possible that it may cure both the CQ-based composite and the CQ-and-TPO based composite better than the polywave, especially at higher depths.

Further studies must be done to determine what effect does the transmission and curing profile have on polymerization of the composite deep in the cavity and whether the predictions previously stated are true. Furthermore, it should be tested whether beam homogeneity has any effect on how homogenous the cure is in bulk fill composites. This is especially important for the polywave, which has higher beam inhomogeneity than the monowave, with different spectral and irradiance hotspots located over different areas in 
the composite. For this reason the overall objective of further studies is to compare the homogeneity of cure into the cavity of a resin restoration for different bulk composites with varying photo initiators cured by polywave or monowave LCUs. The homogeneity of cure will be determined by comparing the degree of conversion at various depths and locations within the cavity using an FTIR.

\section{Acknowledgements}

I want to thank Dr. Jack Ferracane and Dr. Dayane Oliveira for all their help, support, and guidance on this project. Without them this project would not have been possible.

\section{Bibliography}

1. Arikawa, H., Kanie, T., Fujii, K., Takahashi, H., \& Ban, S. (2008). Effect of Inhomogeneity of Light from Curing Units on the Surface Hardness of Composite Resin. Dental Materials Journal , 21-28.

2. Ciba. (2003). Photoinitiators for UV Curing. Key Products Selection Guide 2003 . Ciba Specialty Chemicals.

3.David, J. R., Gomes, O. M., Gomes, J., Loguercio, A., \& Reis, A. (2007). Effect of exposure time on curing efficiency of polymerizing units equipped with light-emitting diodes. Journal of Oral Science, 19-24.

4.DENSPLY. (2013, 07 25). Smartlite Focus. Milford, DE.

5.Ferracane, J. L., Mitchem, J., Condor, J., \& Todd., R. (1997). Wear and marginal breakdown of composites with various degrees of cure. Journal of Dental Research, 1508-1516.

6.Ivoclar Vivadent. $(2012,12)$. An extraordinary family of curing lights. Amherst, NY.

7.Ivoclar Vivadent. (2014, 3). Scientific Documentation Tetric EvoCeram Bulk Fill. . Liechtenstein.

8.Kamoun, E. A., Winkel, A., Eisenburger, M., \& Menzel, H. (2014). Carboxylated camphorquinone as visible-light photoinitiator for biomedical application: Synthesis, characterization, and application. Arabian Journal of Chemistry .

9.Kerr Corporation. (2012, 07 17). SonicFill Sonic-Advanced, Bulk Fill Composite. Portfolio of Scientific Research . 
10.Michaud, P.-L., Price, R., Labrie, D., Rueggeberg, F., \& Sullivan, B. (2014).

Localised irradiance distribution found in dental light curing units. Journal of Dentistry, 129-139.

11.Miletic, V., \& Santini, A. (2012). Micro-Raman spectroscopic analysis of the degree of conversion of composite resins containing different initiators cured by polywave or monowave LED units. Journal of Dentistry , 40, 106-113.

12.Nomoto, R. (1997). Effect of light wavelength on polymerization of light cured resins. Dental Materials Journal , 60-73.

13.Nomoto, R., Uchida, K., \& Hirasawa, T. (1994). Effect of light intensity on Polymerization of Light-cured Composite Resins. Dental Materials Journal , 198-205.

14.Price, R., Labrie, D., Rueggeberg, F., Sullivan, B., Kostylev, I., \& Fahey, J. (2014). Correlation between the beam profile from a curing light and the microhardness of four resins. Dental Materials .

15.Sigma Aldrich. "Application: Free Radical initiators." Sigma Aldrich. https://www.sigmaaldrich.com/content/dam/sigma-

16.Yearn, J. (1985). Factors affecting cure of visible light activated composites. International Dental Journal , 35 (3), 218-225. 